\title{
Depuración de aguas residuales por procesos de membrana. Caso de una industria petroquímica
}

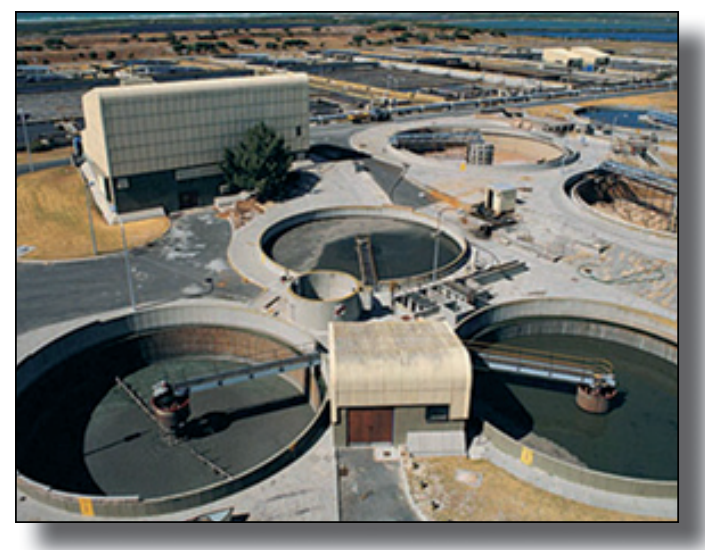

Jaime Lora-Garcia*

Jose Miguel Arnal-Arnal*

Fernanda López-Pérez *

$M^{0}$ Carmen León-Hidalgo*
Dr. Ingeniero Industrial

Dr. Ingeniero Químico

Dra. Ciencias Químicas

Dra. Ingeniería y Producción Industrial

* UNIVERSITAT POLITĖCNICA DE VALÈNCIA. ESSII. Dpto. de Ingeniería Química y Nuclear. Camino de Vera, $s / n-46022$ Valencia. Tfno: +34963877 633. XXXXXXXXXXXXX

** UNIVERSITAT POLITÈCNICA DE VALĖNCIA.EPSA. Placa Ferràndiz i Carbonell, $\mathrm{s} / \mathrm{n}$ -

03801 Alcoy (Alicante) Ifno: +34 966528 470. jora@iqn.upv.es

Recibido: 23/02/2012 • Aceptadd: 09/07/2012

D0l: http://dx.doi.org/10.6036/4689

\section{WASTEWATER TREATMENT BY MEANS OF MEMBRANE PROCESSES.}

\section{A CASE STUDY OF PETROCHEMICAL INDUSTRY}

\section{ABSTRACT}

- This paper studies the treatment of secondary effluent from a wastewater treatment plant of a petrochemical industry by using reverse osmosis (RO) for wastewater reclamation. The petrochemical industry requires a large amount of water with a conductivity less than $100 \mathrm{pS} / \mathrm{cm}$ for use in several production processes. The water from the wastewater treatment plants stations of this type of industry has a high conductivity $(>5000 \mathrm{\mu S} / \mathrm{cm})$, and therefore is not suitable for reuse. A possible solution is a specific treatment (tertiary treatment) by membrane technologies.

The proposed system considers an initial filtration step to remove suspended solids and colloidal matter. Then the effluent is introduced to a reverse osmosis system, which operates at low pressure in two stages.

The methodology used includes two sections. On the one hand the experimental tests to define the critical parameters of the membrane process design, and on the other the scale of the industry level to estimate the costs of the proposed treatment. This system has succeeded in recovering $75 \%$ of the effluent, as water with sufficient quality for reuse $(<100 \mathrm{mS} / \mathrm{cm})$. The cost of reclaimed water $(0.76 / \mathrm{m} 3)$ allows to consider the industrial implementation of the process.

- Key Words: non-conventional water resources, reuse, reclaimed water, membrane processes, reverse.

\section{RESUMEN}

El presente trabajo estudia el tratamiento del efluente secundario de una estación depuradora de aguas residuales de una industria petroquímica mediante ósmosis inversa (OI) para su reutilización.

La industria petroquímica requiere una gran cantidad de agua con una conductividad inferior a $100 \mu \mathrm{S} / \mathrm{cm}$ para su uso en varios procesos de producción. El agua de las estaciones depuradoras de aguas residuales de este tipo de industrias presenta una elevada conductividad (>5000 $\mu \mathrm{S} / \mathrm{cm}) \mathrm{y}$, por tanto, no es apta para ser reutilizada. Una solucion posible es un tratamiento especifico (tratamiento terciario) mediante tecnologías de membranas.

El sistema propuesto considera una etapa de filtración inicial para eliminar los sóli- dos en suspensión y la materia coloidal. A continuación, el efluente se introduce a un sistema de ósmosis inversa, que trabaja a baja presión en dos etapas.

La metodología utilizada comprende dos apartados: por una parte, los ensayos experimentales para definir los parámetros críticos del diseño del proceso de membranas y, por otra, el escalado a nivel industrial para estimar los costes del tratamiento propuesto.

Mediante este sistema se ha conseguido recuperar un $75 \%$ del efluente como agua con suficiente calidad para su reutilización $(<100 \mu \mathrm{S} / \mathrm{cm})$. El coste del agua recuperada $\left(0.76 € / \mathrm{m}^{3}\right)$ que permite considerar la implantación industrial del proceso.

Palabras Clave: recursos hídricos no convencionales, 
reutilización, agua recuperada, procesos de membrana, osmosis inversa.

\section{INTRODUCCIÓN}

El agua es un elemento esencial para mantener la vida. Sabemos que el desarrollo social se basa en la disponibilidad de agua segura, y el desarrollo económico requiere recursos energéticos y actividades industriales que dependen del agua. La vulnerabilidad creciente de los recursos hídricos del planeta, tanto en disponibilidad como en calidad, necesita objetivos y actuaciones que permitan, en un horizonte no muy lejano, poder administrar eficazmente tan preciado recurso.

Las diferentes soluciones planteadas se pueden agrupar en dos contextos. En el plano socio-político destacan la gestión racional de la demanda, la política institucional sobre el uso responsable del agua y las políticas de tarifas eficientes. En el apartado tecnológico cabe citar la gestión eficiente de los recursos existentes, los trasvases de cuencas y la desalación y reutilización de las aguas depuradas. Estas últimas constituyen no solo una herramienta para la gestión eficaz del denominado ciclo integral del agua, sino que representan una fuente de recursos hídricos no convencionales (Figura 1).

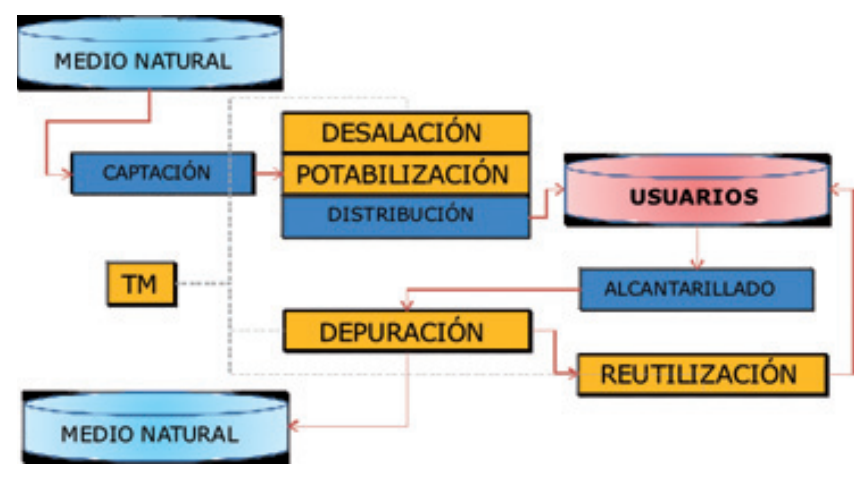

Figura 1: La tecnología de membranas (TM) en el ámbito del ciclo integral del agua

La aplicación de la tecnología de membranas ha transformado la forma de abordar los tratamientos del agua en los últimos años gracias a su potencial separador específico que permite la eliminación de la contaminación en función de su tamaño y naturaleza química. Las propiedades permeselectivas de las membranas y la aplicación de una fuerza impulsora basada en gradientes de presión o potencial eléctrico, permiten la eliminación total o parcial de un gran número de sustancias presentes en las aguas residuales o en las fuentes naturales salinizadas. En la Tabla 1 se resumen las características más importantes de los procesos de membranas que se utilizan en el ciclo integral del agua.

Los efluentes industriales representan un potencial de recursos hídricos muy importante. Un ejemplo de ello son los datos sobre el consumo de agua en los sectores manufactureros y el bajo nivel de reutilización de los mismos, tal como se muestra en la Tabla 2.

\begin{tabular}{|l|r|l|}
\hline Suministro de agua red y captación propia & 1350 & \\
\hline Aguas residuales vertidas & 908 & \\
\hline Agua reutilizada & 114 & $8,1 \%$ \\
\hline
\end{tabular}

La mayor parte de las industrias con gran producción de efluentes tienen implantado un sistema de depuración de sus aguas residuales. Sin embargo, para adaptar el agua depurada a su reutilización en la industria se requiere un tratamiento terciario acorde con las directrices del RD 1620/2007. Entre las mejores técnicas disponibles, la tecnología de membranas permite obtener agua de calidad a un coste aceptable. Esto es debido a su bajo consumo energético, compacidad, modularidad y facilidad de operación. En concreto, la tecnología de OI es la más utilizada para la recuperación de agua con una elevada concentración de sólidos totales disueltos (STD) [1,2].

En el caso concreto de la industria estudiada, un caudal de agua considerablemente elevado es vertido al mar directamente desde su estación depuradora de aguas residuales industriales, que emplea un tratamiento biológico. Ante la escasez de recursos hídricos de la zona y la necesidad de ampliar el proceso productivo, se pensó en un tratamiento de estos efluentes mediante ósmosis inversa como la técnica

\begin{tabular}{|c|c|c|c|}
\hline PROCESO & $\begin{array}{l}\text { FUERZA } \\
\text { IMPULSORA }\end{array}$ & $\begin{array}{l}\text { RANGO DE } \\
\text { TAMAÑO }\end{array}$ & PATÓGENOS Y COMPUESTOS ELIMINADOS \\
\hline Microfiltración (MF) & Gradiente de presión & $1-0.1 \mu \mathrm{m}$ & Turbidez, bacterias, algas, protozoos \\
\hline $\begin{array}{l}\text { Ultrafiltración (UF) } \\
\text { Bioreactores de membrana (MBR) }\end{array}$ & Gradiente de presión & $0.1 \mu m-1.0 \mathrm{~nm}$ & Virus, polisacáridos, asbestos \\
\hline Nanofiltración (NF) & Gradiente de presión & \multirow[b]{2}{*}{$<1.0 \mathrm{~nm}$} & \multirow{2}{*}{$\begin{array}{c}\text { Dureza, color, iones metálicos, urea, ácidos } \\
\text { orgánicos, }\end{array}$} \\
\hline Electrodiálisis Reversible (EDR) & $\begin{array}{l}\text { Gradiente de } \\
\text { potencial eléctrico }\end{array}$ & & \\
\hline Osmosis inversa (OI) & Gradiente de presión & $<100$ Dalton & Iones salinos \\
\hline
\end{tabular}

Tabla 1: Procesos de membrana en el ciclo integral del agua 
más adecuada para obtener agua de calidad suficiente. Los efluentes de este tipo de industria petroquímica presentan elevados valores carga contaminante sobretodo de grasas, DQO, COT, así como grandes cantidades de sólidos totales disueltos y bajas concentraciones de sólidos en suspensión. Además, los valores de $\mathrm{pH}$ suelen situarse por encima del valor de 7 [3].

Las exigencias del agua de proceso $(<100 \mu \mathrm{S} / \mathrm{cm})$ hacen que, pese a realizarse pretratamientos adecuados, el tratamiento terciario mediante

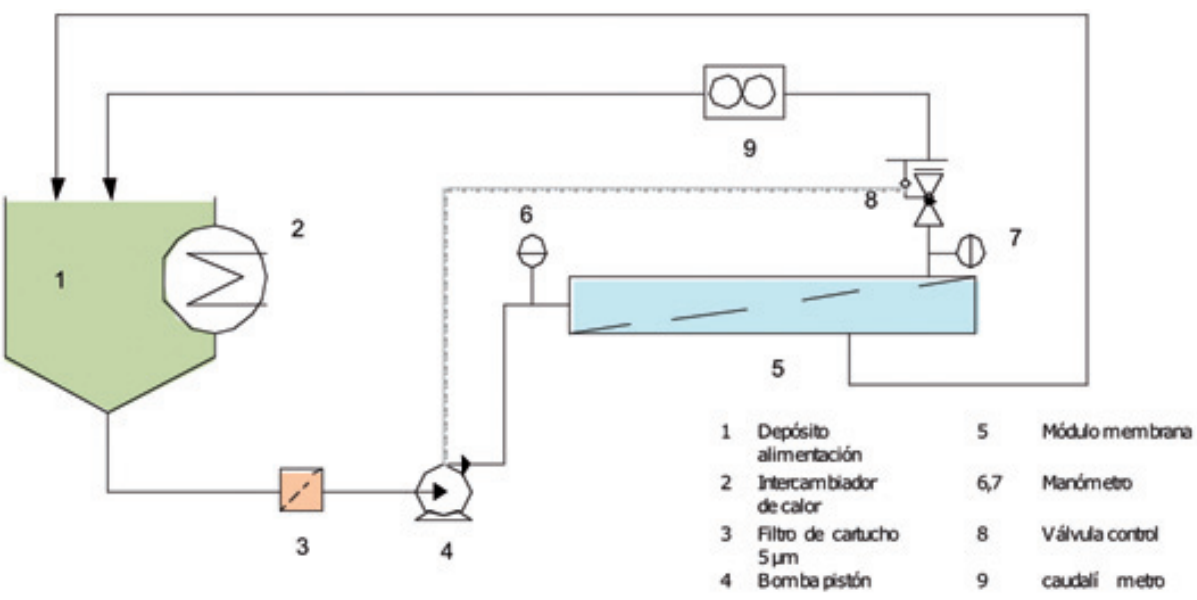

Figura 2: Planta piloto para los ensayos experimentales de 01 OI de baja presión de las aguas residuales pueda no resultar suficiente. Es por ello que en el presente trabajo se ha decidido realizar por un lado el tratamiento terciario del agua residual mediante OI, y por otro realizar un tratamiento de OI al agua de suministro municipal, de manera que la mezcla de los dos permeados cumpla las exigencias de calidad $[4,5]$.

\section{MATERIAL Y MÉTODOS}

Para la realización de los experimentos, se utilizó agua proveniente del tratamiento secundario de la planta de tratamiento de aguas residuales de una empresa del sector petroquímico. Este efluente se caracteriza por una elevada conductividad, comprendida entre 5000-8500 $\mu \mathrm{S} / \mathrm{cm}$, una concentración en STD entorno a $5200 \mathrm{mg} / \mathrm{L}$ y un $\mathrm{pH}$ en el rango 7.0 - 8.6, tal como se resume en la Tabla 3. Por otra parte, el agua de suministro municipal presenta una conductividad promedio de $1400 \mu \mathrm{S} / \mathrm{cm}$, un $\mathrm{pH}$ de 7.8 y una concentración de STD de $1500 \mathrm{mg} / \mathrm{L}$.

\begin{tabular}{|l|l|l|l|}
\hline & \multicolumn{2}{l|}{ EFLUENTE } & \multicolumn{2}{l|}{$\begin{array}{l}\text { AGUA } \\
\text { MUNICIPAL }\end{array}$} \\
\hline $\mathrm{pH}$ & & 8.2 & 7.0 \\
\hline $\begin{array}{l}\text { Sólidos en } \\
\text { suspensión }\end{array}$ & $\mathrm{mg} / \mathrm{L}$ & 60 & 25 \\
\hline Conductividad & $\mathrm{\mu S} / \mathrm{cm}$ & $\begin{array}{l}5000- \\
8770\end{array}$ & $800-1700$ \\
\hline Temperatura & ${ }^{\circ} \mathrm{C}$ & 25 & 25 \\
\hline SDI (15 $\mathrm{min})$ & & $>5$ & $>5$ \\
\hline STD & $\mathrm{mg} / \mathrm{L}$ & 5200 & 1500 \\
\hline Dureza Total & $\mathrm{mg} / \mathrm{LC} \mathrm{CaC} 03$ & 38 & 26 \\
\hline SO4= & $\mathrm{mg} / \mathrm{L}$ & 2500 & 650 \\
\hline DQ0 & $\mathrm{mg} / \mathrm{L}$ & 133 & 12 \\
\hline
\end{tabular}

Tabla 3: Características de las diferentes alimentaciones tratadas

En todos los ensayos de OI se realizó un pretratamiento con diferentes niveles de filtración $(200-20 \mu \mathrm{m})$ como paso previo para reducir los valores de SDI (silt density index) de las corrientes tratadas. Con ello se trató de minimizar el potencial de ensuciamiento de las membranas de OI.

Los diferentes ensayos se realizaron en una planta piloto diseñada y construida en el Departamento de Ingeniería Química y Nuclear de la UPV, cuyo esquema se presenta en la Figura 2. El modo de operación es en recirculación total, de modo que las corrientes de permeado y concentrado retornan a un tanque de alimentación (1) de $60 \mathrm{~L}$ de capacidad, para mantener constante la concentración. La alimentación es impulsada al modulo de membrana con una bomba de pistones (4) a través de un sistema de filtración de cartuchos de $5 \mu \mathrm{m}$ (3). El flujo de concentrado y la presión transmembranal se mantienen constantes mediante la válvula de regulación (4) y el variador de frecuencia del

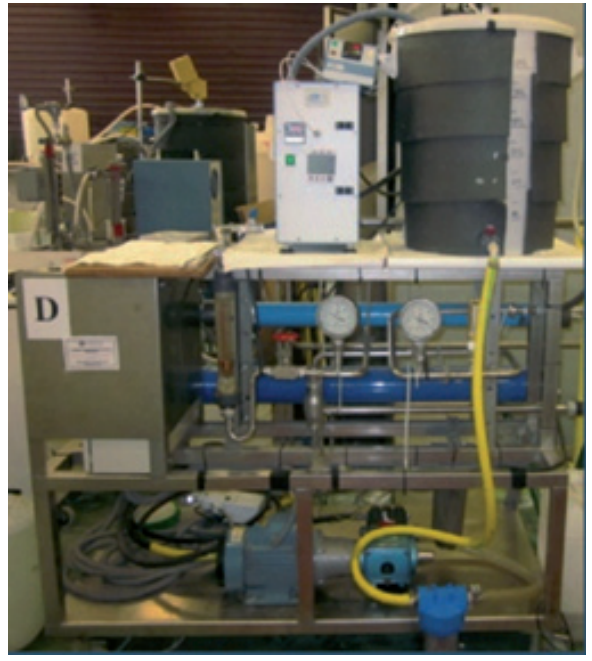

Figura 3: Disposición de los módulos de membrana en la planta piloto para ensayos experimentales de 01 


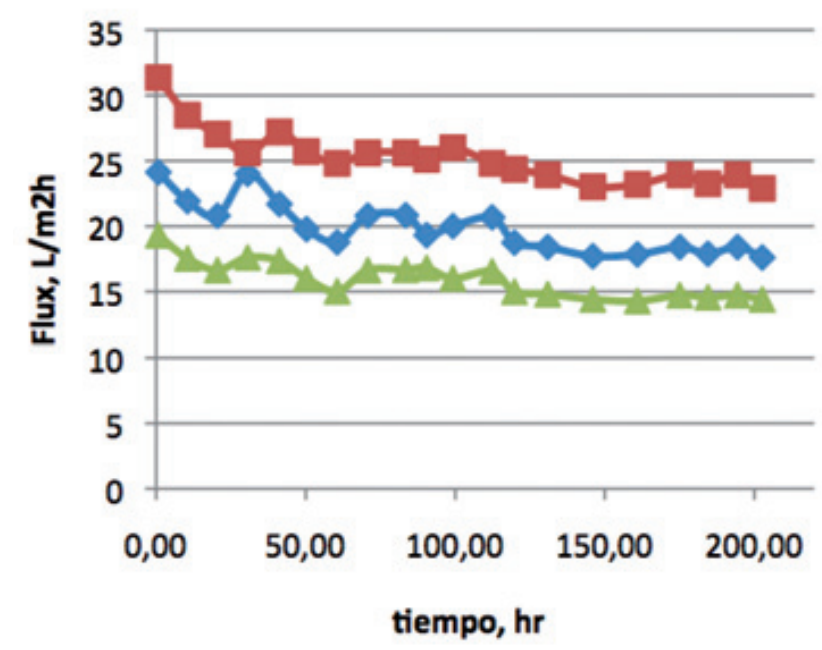

Figura 4: Evolución del flux de permeado obtenido a partir del agua municipal

motor de la bomba. La temperatura se mantiene constante a $25{ }^{\circ} \mathrm{C}$ gracias a un sistema de intercambio de calor (2).

La variable seleccionada para el estudio del comportamiento del sistema fue la presión transmembranal en el intervalo de 0.5 a $1.5 \mathrm{MPa}$, ajustando el caudal de alimentación para mantener una conversión del proceso en el 15\%. Los módulos de membrana utilizados en los ensayos experimentales de OI, fueron del tipo CPA4 y LFC3 de 2.5" de la firma Hydranautics. Su capa activa es de poliamida aromática con un rechazo al $\mathrm{NaCl}$ superior al 99.5\% [6]. En la Figura 3 se puede observar la disposición de ambos módulos en la planta piloto.

Para el tratamiento del efluente secundario se seleccionaron las membranas que ofrecen una mayor resistencia al ensuciamiento LFC3, mientras que los ensayos con agua municipal se llevaron a cabo con las membranas CPA4. Para diferenciar las diferentes calidades del agua, se define calidad A y calidad B para los permeados obtenidos a partir del agua municipal y el efluente secundario respectivamente.

En una segunda fase se realizó una simulación ajustada a los resultados experimentales mediante el software IMSDesign de Hydranautics. Finalmente a partir de los resultados obtenidos se estimaron los costes del agua recuperada a fin de establecer la viabilidad del proceso propuesto.

\section{RESULTADOS}

En primer lugar se muestran los resultados obtenidos en los diferentes procesos de OI desde el punto de vista de la capacidad separadora de las membranas. En la Tabla 4 se muestra las características más significativas del permeado de OI obtenido a partir del efluente secundario y del agua municipal respectivamente.

\begin{tabular}{|l|c|r|r|}
\hline \multicolumn{1}{|c|}{ Parámetro } & Unidades & $\begin{array}{c}\text { Agua } \\
\text { Calidad B }\end{array}$ & $\begin{array}{c}\text { Agua } \\
\text { Calidad A }\end{array}$ \\
\hline $\mathrm{pH}$ & & 6.15 & 6.14 \\
\hline Turbidez & $\mathrm{NTU}$ & 0 & 0 \\
\hline Conductividad & $\mu \mathrm{S} / \mathrm{cm}$ & 153 & 12 \\
\hline Temperatura & ${ }^{\circ} \mathrm{C}$ & 25 & 25 \\
\hline COT & $\mathrm{mg} / \mathrm{L}$ & $<1$ & $<1$ \\
\hline SDI $(15 \mathrm{~min})$ & & 0 & 0 \\
\hline SDT & $\mathrm{mg} / \mathrm{L}$ & 104 & 8 \\
\hline
\end{tabular}

Tabla 4: Características del permeado de 01 de las corrientes tratadas

Desde el punto de vista de la permeabilidad de las membranas, en la Figura 4 se presentan los resultados de la evolución del flux con el tiempo, en función de las diferentes presiones ensayadas para el caso del agua municipal. En el caso del efluente secundario, en la Figura 5 se muestran los resultados obtenidos para la presión de 1,0 $\mathrm{MPa}$.

Acontinuación, se muestran los resultados de la simulación del proceso a escala industrial ajustada a los resultados experimentales. Para el efluente secundario se planteó un sistema de OI de dos etapas con un caudal de alimentación de $175 \mathrm{~m} 3 / \mathrm{h}$, bar y una conversión total del $75 \%$. El diseño resultante requiere una configuración de 26 tubos de presión (6 módulos por tubo de 8") en la primera etapa, y 12 tubos de presión en la segunda etapa, con una presión de alimentación de 9.5 bar. Para el caso del agua municipal las condiciones de operación fueron: caudal de alimentación de $273 \mathrm{~m} 3 / \mathrm{h}$ y una conversión total del $80 \%$. En

Figura 5: Evolución del flux de permeado obtenido a partir del efluente secundario a la presión transmembranal de 1,0 MPa 


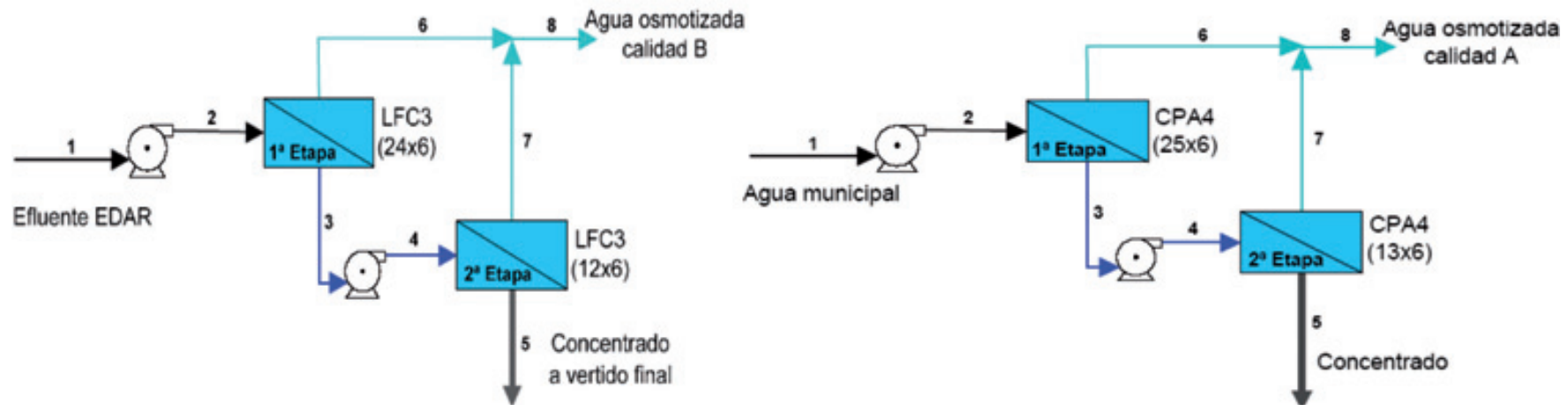

Figura 6: Esquema de las configuraciones resultantes de la simulación d proceso de 01

este caso la configuración comprende 25 tubos de presión (6 módulos por tubo) en la primera etapa, y 13 tubos de presión en la segunda etapa, con una presión de alimentación de 15.8 bar. En la Figura 5 se muestra un esquema de las configuraciones obtenidas.

Finalmente en la Tabla 5 se presentan los valores de los parámetros más relevantes de la mezcla de los dos tipos de permeado obtenidos en una relación de 2:3.

\begin{tabular}{|l|c|r|}
\hline \multicolumn{1}{|c|}{ Parámetro } & Unidades & Mezcla 2:3 \\
\hline $\mathrm{pH}$ & & 6.29 \\
\hline Turbidez & $\mathrm{NTU}$ & $<1$ \\
\hline Conductividad & $\mu \mathrm{S} / \mathrm{cm}$ & 66 \\
\hline COT & $\mathrm{mg} / \mathrm{L}$ & 0 \\
\hline SDI $(15 \mathrm{~min})$ & & 44 \\
\hline STD & $\mathrm{mg} / \mathrm{L}$ & 0 \\
\hline
\end{tabular}

Tabla 5. Características de la mezcla de permeados

\section{DISCUSIÓN}

En primer lugar se puede comprobar en la Tabla 4 que la capacidad separadora del sistema propuesto permite obtener corrientes de permeados libres de materia en suspensión y materia orgánica disuelta, así como una reducción considerable ( $>98 \%$ ) de sales disueltas.

El análisis de la permeabilidad de las membranas utilizadas en cada caso (Figuras 4 y 5), manifiesta diferentes comportamientos frente al ensuciamiento inevitable de las membranas. En el caso del agua municipal, una vez alcanzado el flux estable, las variaciones del mismo son prácticamente despreciables. Dicho valor está en consonancia con el flux nominal del fabricante $\left(\mathrm{L} / \mathrm{m}^{2} \mathrm{~h}\right.$ a $\left.1,5 \mathrm{MPa}\right)$. Sin embargo, cuando se trata el efluente del secundario, a pesar de la etapa de pre-tratamiento considerada, se produce un nivel de ensuciamiento significativo que requiere un protocolo de limpieza periódico, aproximadamente cada 3 o cuatro días durante 30 minutos, para mantener un flux estable en torno a $18 \mathrm{~L} / \mathrm{m}^{2} \mathrm{~h}$.

Se consideró que la causa del ensuciamiento era fundamentalmente por materia orgánica procedente el tratamiento biológico del secundario. Por ello, el proceso de limpieza propuesto consistió en aplicar una disolución alcalina $(0,03 \mathrm{M} \mathrm{NaOH})$ en modo "flush", es decir por el lado de la alimentación, y a continuación, un enjuague con agua osmotizada. Con ello se conseguía mantener el flux estable durante largos periodos operativos.

Tal como se observa en la Tabla 4, tras el tratamiento terciario del efluente secundario mediante OI el agua obtenida no presenta la calidad exigida. Es por ello que es necesario mezclar el agua osmotizada de dicho sistema con el agua osmotizada del sistema de OI generada a partir del agua municipal. A partir de una relación de mezcla 2:3 los valores de la tabla 5 indican que el agua obtenida si alcanza el requerimiento exigido para su reutilización en la planta petroquímica (Conductividad $<100 \mu \mathrm{S} / \mathrm{cm}$ ).

Las pruebas de simulación requieren una serie de ajustes para adaptar las diferentes configuraciones a los valores de flux estable obtenidos en las pruebas descrestas anteriormente. De esta forma, a pesar de que el software daba resultados mucho más competitivos desde el punto de vista económico, se opto por encontrar soluciones mas conservadoras para tener en cuenta el riesgo de ensuciamiento en el caso del efluente secundario, trabajando a un flux más reducido que el nominal del fabricante $\left(40 \mathrm{~L} / \mathrm{m}^{2} \mathrm{~h}\right)$.

Una vez analizados los resultados se realizó un estudio de los costes de explotación del tratamiento del sistema de OI propuesto, considerando que se trataba de un sistema complementario a las instalaciones de tratamiento de las aguas residuales de la empresa. Por consiguiente se estimó una reducción por sinergias de determinados costes (personal, mantenimiento, y servicios) sobre los costes globales.

El coste global de la inversión estimado para este tipo de planta rondaría los 5,4 $\mathrm{M} €$. El coste de producción del agua reutilizada alcanzaría los $0.76 € / \mathrm{m}^{3}$, aunque dicho coste 
es susceptible de minorarse considerando el coste del agua ahorrada así como el correspondiente a la minimización de residuos y canon de vertido. Por otra parte, conviene resaltar que en este tipo de procesos existe una fuerte dependencia de la energía y de los intereses de financiación sobre los costes estimados anteriormente.

\section{CONCLUSIONES}

De los resultados obtenidos en el presente estudio se derivan dos cuestiones principales. Por una parte, se constata la viabilidad técnica de los procesos de membrana en cuanto a su integración en el tratamiento de las aguas residuales industriales $\mathrm{y}$, por otra, permiten obtener un elevado porcentaje de recuperación del agua tratada como agua de proceso.

El sistema estudiado para la reutilización de aguas de una empresa del sector petroquímico comprende dos líneas de tratamiento: una para el efluente secundario de la salida de la estación depuradora y otra para el tratamiento del agua municipal. Mediante este sistema cada una de las líneas dispone de los pre-tratamientos requeridos para la aplicación de la OI en función del agua procesada, bien sea de origen industrial o municipal.

Las experiencias en planta piloto han permitido establecer tanto los valores del flux estable necesario para el diseño de la instalación industrial, y las consideraciones relativas al ensuciamiento de las membranas en cada uno de los casos estudiados.

Para el caso del tratamiento del agua municipal se consideran adecuadas las membranas baja presión del tipo CPA4 de Hydranautics, y los datos obtenidos sobre el flux estable son muy similares al aportado por el fabricante. En el caso del tratamiento del efluente secundario es necesario trabajar con membranas de bajo ensuciamiento tipo LFC3 y con valores de flux prácticamente al 50\% del nominal.

Los resultados alcanzados muestran que se puede recuperar agua residual con una calidad adecuada para su uso como agua de proceso (conductividad inferior a los 100 $\mu \mathrm{S} / \mathrm{cm})$.

La empresa estudiada vierte aproximadamente unos $175 \mathrm{~m}^{3} / \mathrm{h}$ de agua residual depurada al mar. Mediante el tratamiento terciario del agua residual pueden obtener cerca de $130 \mathrm{~m}^{3} / \mathrm{h}$ de agua tratada lo que representa una recuperación del 75\%. De esta manera, un 37.5\% del agua de proceso tiene su origen en las aguas residuales generadas en la propia empresa.

El coste del agua recuperada se estima en $0.76 € / \mathrm{m}^{3}$, sin considerar una serie de cuestiones como el ahorro en el consumo de agua de suministro municipal. Adicionalmente, mediante la reutilización se reduce el volumen de vertidos lo que repercute en un ahorro por la reducción del canon de saneamiento, así como el consiguiente beneficio medioambiental.

\section{AGRADECIMENTOS}

El presente Estudio ha sido desarrollado al amparo del Convenio de Colaboración entre el la Empresa fomento Agrícola Castellonense (FACSA) y el Instituto de Seguridad Industrial, Radiofisica y Medioambiente de la Universitat Politècnica de València para el estudio sobre "Sistemas y procesos de optimización de los recursos hídricos en industrias del sector químico".

\section{BIBLIOGRAFÍA}

[1] Gozálvez JM, Sanz D, Lora J, et al. "Nanofiltration of secondary effluent for wastewater reuse in the textile industry". Desalination. 2008, Vol. 222 p. 272-279. http:// dx.doi.org/10.1016/j.desal.2007.01.173

[2] Wilf Mark, Alt Steven. "Application of low fouling RO membrane elements for reclamation of municipal wastewater". Desalination. 2000, Vol. 132 p. 11-19. http:// dx.doi.org/10.1016/S0011-9164(00)00130-2

[3] Raetens D. "Water pinch analysis: minimization of water and wastewater in the process industry" en "Water recycling and resource recovery in industry". London, IWA Publishing. 2002, p. 203-251.

[4] Gozálvez JM, Sanz D, Lora J, et al. "Estudio preliminar de ultrafiltración mediante membranas de bajo corte molecular como pretratamiento para la reutilización de un agua residual petroquímica" En: Actas del VI Congresso IberoAmericano em Ciências e Tecnologia de Membrana. Campina Grande - Paraiba, Brasil (2007).

[5] Lora J, Sanz D, Gozálvez JM, et al. "Ultrafiltration and nanofiltration pre-treatment of reverse osmosis to reuse wastewater in a petrochemical industry". En: Actas del IDA World Congress-Maspalomas, Gran Canaria- España (2007).

[6] Hydranautics. Ficha Técnica Membrana LFC3 [en línea]. Applied Membranes. [Vista, USA]. Disponible en la Web: <http://www.appliedmembranes.com/pdf/Hydranautics $\% 20$ Elements/LFC3.pdf> [Consulta 2 de Febrero 2011]

[7] Hydranautics. Ficha Técnica Membrana CPA4 [en línea]. Applied Membranes [Vista, USA]. Disponible en la Web:<http://www.appliedmembranes.com/pdf/ Hydranautics\%20Elements/CPA4.pdf> [Consulta 2 de Febrero 2011] 